\title{
The One2I Technique: An Individualized Treatment for Glabellar Lines Based on Clinical and Anatomical Landmarks
}

This article was published in the following Dove Press journal: Clinical, Cosmetic and Investigational Dermatology

\author{
Carla de Sanctis Pecora' \\ Maria Valéria Bussamara \\ Pinheiro' \\ Karin Ventura Ferreira' \\ Gisele Jacobino de Barros \\ Nunes' \\ Hélio Amante Miot $\mathbb{1 D}^{2}$ \\ 'Dermatologie - Clinica, Cirurgia, \\ Cosmiatria e Laser, São Paulo, SP, Brazil; \\ ${ }^{2}$ Departamento de Dermatologia da \\ FMB-Unesp, Botucatu, SP, Brazil
}

\section{Video abstract}

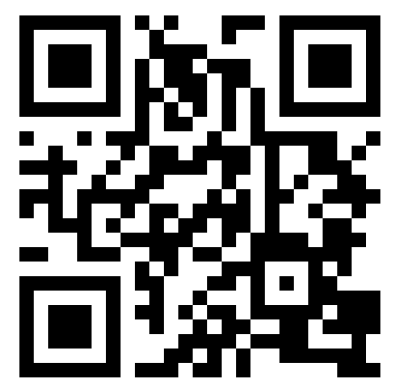

Point your SmartPhone at the code above. If you have a $Q R$ code reader the video abstract will appear. Or use: https://youtu.be/SLAAVhtfrwl
Correspondence: Carla de Sanctis Pecora Dermatologie - Clinica, Cirurgia,

Cosmiatria e Laser, Avenida Ibirapuera,

2907, Conj 90I, São Paulo, SP, CEP

04029-200, Brazil

$\mathrm{Tel} / \mathrm{Fax}+55 \mathrm{I} I 50420872$

Email carla@dermatologie.com.br
Purpose: Botulinum toxin type A is an effective treatment for glabellar dynamic wrinkles. As the muscular group involved in the contraction of the glabella varies among people, individualized treatment can achieve optimal results. This study evaluates a customized assessment for the treatment of glabellar lines with Incobotulinumtoxin-A, leading to an individualized points distribution and dosage.

Patients and Methods: A single-center, evaluator-blinded, therapeutic cohort study enrolled 130 women with moderate or severe glabellar wrinkles. They underwent Incobotulinumtoxin-A injection following the standard 5-point injection at the glabellar muscles $(n=65)$ or an individualized assessment and scheme treatment based on anatomical references of contraction, the One 21 technique $(n=65)$. All the patients were photographed under maximum contraction before treatment (T0) and after 4 weeks (T28). The photos were randomly assessed by two blinded, experienced raters to consensually grade the severity according to the Merz Aesthetics Scales (MAS). The primary clinical efficacy was defined as a 2-point reduction in the MAS score, on Day 28.

Results: The groups were homogeneous regarding age, phototype, and baseline MAS scores. On Day 28 (T28), 64 (98.5\%) patients from the One21 group and 52 (80\%) from the 5-point group reduced the MAS score by at least two points $(p<0.01)$. When adjusted by age and phototype, both groups reduced the MAS score at T28 $(p<0.01)$; nevertheless, patients from the One21 group significantly performed better $(p<0.01)$, with a much higher rate response rate at $\mathrm{T} 28$, in comparison to the 5-point group. Of patients enrolled in the 5-point group, $83.1 \%$ utilized muscle groups other than the procerus and corrugator in glabellar wrinkle formation, and $17(24.6 \%)$ presented asymmetrical contraction. Patients from the 5-point group with glabellar asymmetry and those who utilized the frontalis and orbicularis presented inferior performance $(p<0.05)$, reinforcing the importance of an individualized assessment and treatment plan.

Conclusion: The One21 technique yielded better results than the standard 5-point treatment in reducing glabellar dynamic lines with Incobotulinumtoxin-A, especially for asymmetric lines of the glabella or the involvement of muscle groups other than the procerus and corrugator.

Keywords: botulinum toxin type A, incobotulinumtoxinA, evaluation studies as a topic, face, muscle contraction, treatment, glabella

\section{Introduction}

Botulinum toxin type A (BoNT-A) injections were introduced for aesthetic purposes in 1992 when Carruthers et al published their first paper on the treatment of glabellar wrinkles. The past 30 years have seen important improvements concerning botulinum toxin injection techniques and the expansion of its indications., ${ }^{1,2}$ 
Glabellar expression lines result from the interaction of small muscle group contractions. Over time, dynamic lines become static wrinkles, which adversely affect the facial perception of emotions. ${ }^{3,4}$ Therefore, the treatment of glabellar lines is a common request in dermatologic daily practice. ${ }^{5}$

According to seminal studies, glabellar expression lines should be treated through multiple injections of high doses with low volumes of BoNT-A which allows precise placement of the toxin with little spread to non-targeted areas. Two double-blind, multicenter, controlled studies used $4 \mathrm{U}$ of BoNT-A in five injection points: one in the procerus and two in each corrugator, bilaterally. These injections demonstrated suitable results in the overwhelming majority of patients, while transient adverse effects were rare.$^{6-8}$

In 2012, de Almeida et al proposed a classification of the patterns of glabella contraction, describing five main patterns: "U", "V", "converging arrows", "omega," and "inverted omega". This highlighted individual differences in the muscular contraction causing glabellar lines and advanced the thinking on BoNT-A techniques for the treatment of glabellar dynamic lines. ${ }^{9}$

The variation of muscles used in the contraction of the glabella and their intensity can result in different patterns of dynamic lines or an asymmetrical wrinkling, demanding an individualized regimen of treatment. The current study proposes a customized assessment and treatment technique for BoNT-A injection in the glabellar region.

\section{Methods}

A single-center, evaluator-blinded, therapeutic cohort study enrolled women with visible glabellar wrinkles who underwent Incobotulinumtoxin-A treatments with the standard treatment (5 injection points; $4 \mathrm{U}$ per point) or the proposed One21 technique. The protocol was approved by the Institutional Review Board (Faculdade de Medicina de Botucatu/UNESP, Brazil Ethics Committee), and all the patients provided informed consent to participate which included the publication of images. The study was conducted in accordance with the Declaration of Helsinki.

Male patients were not included because they require higher doses of botulinum toxin for the treatment of the glabellar wrinkles, which could lead to a biased analysis.

All the participants originated from a private clinic. Glabellar treatment with Incobotulinumtoxin-A was administered by the authors, and standardized photographic documentation was collected before treatment (T0) and after 4 weeks (T28).
The standard treatments (5-point) were performed between September 2017 and May 2019, while the One21 technique procedures for the glabella were performed between June 2019 and May 2020. Both groups were treated with incobotulinumtoxin-A $\left(\mathrm{Xeomin}^{\circledR}{ }^{\circledR}\right.$, Merz Pharmaceuticals GmbH, Frankfurt am Main, Germany). ${ }^{10}$ The ONE21 technique aims at the treatment of the upper face wrinkles based on a unique structural and functional anatomy, with an individualized and customized approach regarding points distribution and dosage. This technique was called ONE21 because it refers to an individualized assessment, a one-to-one approach.

The criteria for inclusion were female sex and a score $\geq 2$ on the validated semiquantitative wrinkle-grading Merz Aesthetics Scales (MAS) for dynamic glabellar lines (under maximum contraction) at baseline, ${ }^{11}$ blindly assessed by two independent investigators through standardized photography taken before treatment (T0). ${ }^{12}$ Patients who had been injected with botulinum toxin in a period of interval $\leq 6$ months were not included.

\section{Clinical Assessment}

The muscles of the face are functionally classified into two groups: mastication and facial expression. Muscles of mastication have both origin and insertion in the bones. While muscles of facial expression have their origins in the bone, they are inserted in the SMAS and skin, with the exception of the frontalis that originates in the galea aponeurotica and inserts directly in the skin. ${ }^{13,14}$

Analyzing the anatomy of the glabella, it is possible to identify three main muscular groups: the procerus, the corrugator, and the depressor supercilii. ${ }^{15}$ For the analysis of the lines that result from muscle contraction, it is imperative to consider the intersection of those groups with the frontalis and the orbicularis oculi.

Wrinkles are formed as a consequence of the contraction of the underlying muscles of facial expressions, perpendicularly to the muscle fibers. Therefore, the analysis of the wrinkles under maximum contraction allows for inference to the role of each muscular group leading to the individual patterns of glabellar lines. In general, vertical medial lines in the glabella are related to the contraction of the corrugator muscle. Horizontal lines, located at the root of the nose, are formed as a result of the contraction of the procerus, and horizontal parallel lines in the central part of the forehead are related to a combined action of the frontalis. The presence of diagonal lines medially to the medial end of the eyebrow denotes the action of the depressor supercilii. ${ }^{9,16,17}$ Vertical parallel lines 

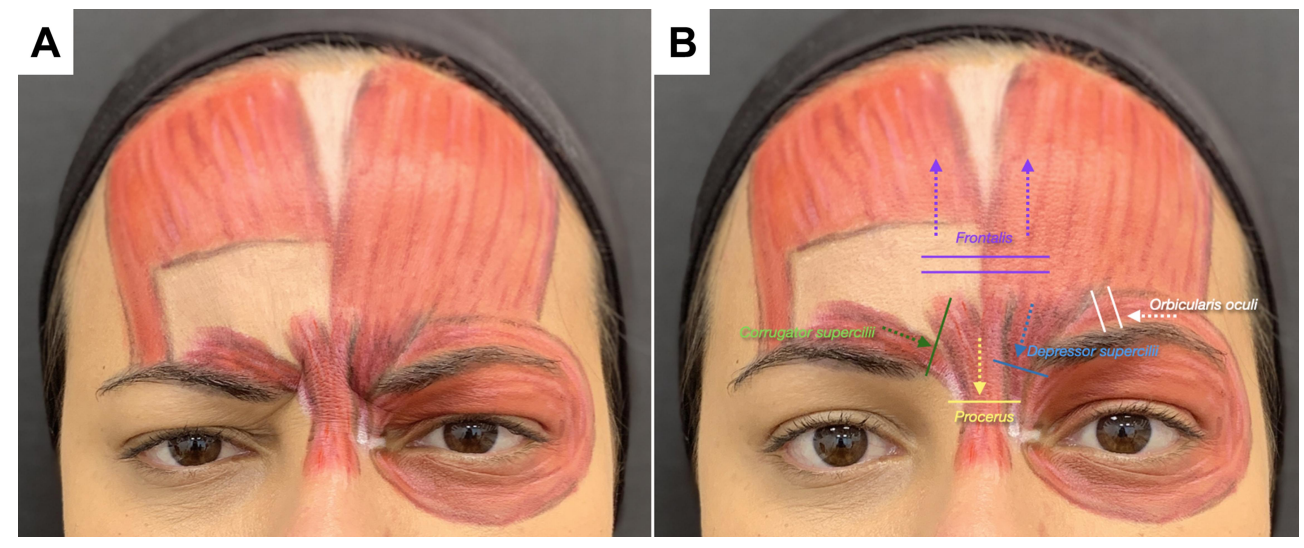

Figure I (A) Representation of muscle groups involved in glabellar frown lines.

(B) Schematic representation of glabellar lines perpendicular to the direction of each underlying muscle.

laterally above the brows indicate the intersection of the corrugator's tail fibers with the orbicularis (Figure 1).

Different patterns of skin lines may be the result of the contraction of muscles with the same attachments and distinctive arrangements of fibers, such as the frontalis, and by muscles having a single origin with three different cutaneous insertions, such as the corrugator supercilii. These muscles run over the medial head of the orbital portion of the orbicularis oculi and are inserted into the deep layer of the skin along the medial half of the eyebrow. Abramo et al describe three symmetrical anatomical patterns for the corrugator supercilii (types I, II, and III) when the muscles are equal on both sides of the glabella and three asymmetrical ones (types IV, V, and VI) when the muscles have distinct types on each side of the glabella. The number, length, and depth of the skin lines depend on the mass and strength of the muscle contraction. ${ }^{18}$

For this assessment, which aimed to identify an individual's glabella pattern, the doctor and patient were positioned face-to-face. The functional anatomy was observed at rest and under maximum contraction, with attention to the distribution and shape of the wrinkles, asymmetries, the presence of eyebrow and eyelid ptosis, and the positioning of the eyebrows as well as the distance between them. Gender, ethnicity, and age were also considered.

\section{Treatment Regimens}

$100 \mathrm{U}$ vial of Incobotulinumtoxin-A was reconstituted in $2 \mathrm{~mL}$ of $0.9 \%$ sterile, non-preserved saline $(50 \mathrm{U} / 1 \mathrm{~mL})$. The treatment was administered by the investigators in the first 20 months following the standard practice of injection (5 injection points; $4 \mathrm{U}$ per point, one injection in the procerus muscle and injections into both the medial and lateral corrugator muscles, bilaterally). In the following 12 months, treatment was administered according to the One21 dosing and point injection map for the glabellar area, determined after individual assessment, varying from 3 to 12 points of injections and addressing the pattern and distribution of the glabellar lines under maximum contraction (Figure 2).
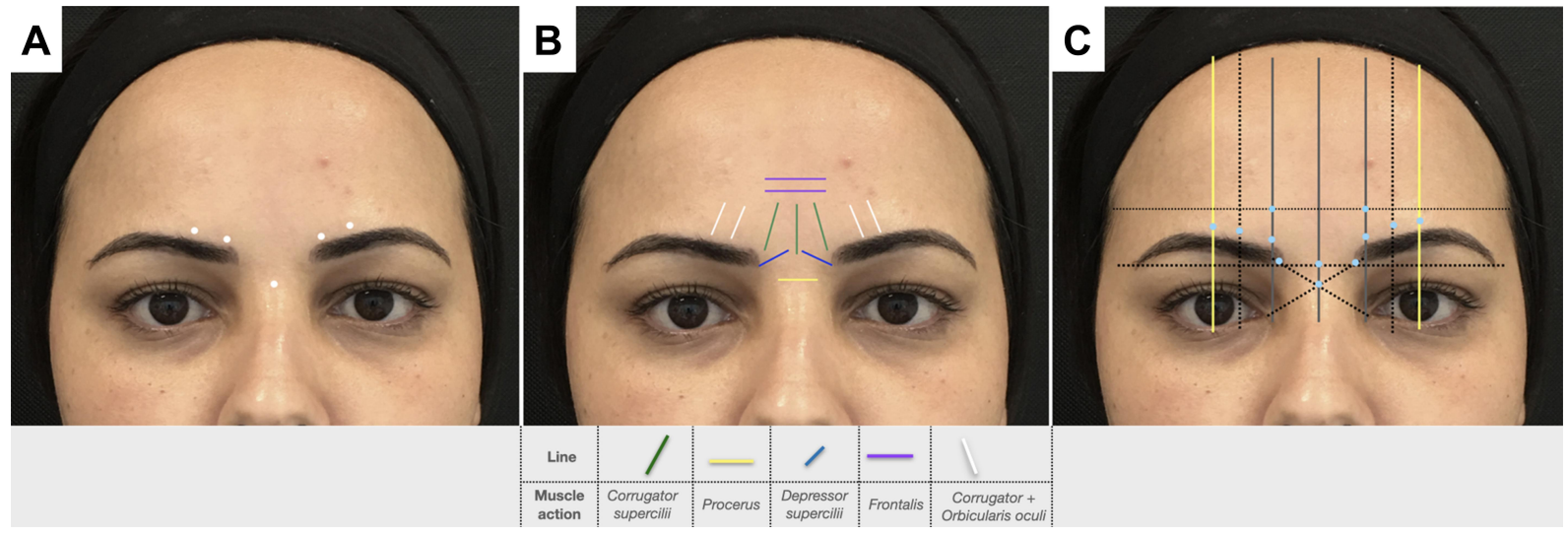

Figure 2 (A) Standard 5-point glabellar technique, focused on the treatment of the procerus and corrugators. (B) Schematic assessment of the glabellar muscles involved in the contraction, according to the pattern of line formation in the One2I technique. (C) Schematic mapping of the One2I technique for treating the procerus, corrugators, orbicularis oculi, depressor supercilii and frontalis. 
The total dosage of Incobotulinumtoxin-A used in the One21 technique was based on the patients' needs, the recommendations published in 2013 by Carruthers et al and in 2015 by Yutscovskaya et al, and the guidelines published by the Global Aesthetics Group in 2016. ${ }^{19-21}$ The positioning of injection points and the depth of injections into the depressor muscles were also considered.

The treatment of each of the predominant muscular groups in the One21 technique is described below.

\section{Corrugators}

At the origin, the injection point was coincident with the line crossing the inner canthus, placed $1 \mathrm{~cm}$ above the orbital rim. The needle was deeply introduced, directed upward. A dose of 4-6 U was administered, according to the mass and strength of the muscle. At the insertion, the injection point was coincident with the line between the inner canthus and mid pupillary line (dotted line). Nearly $30 \%$ of the $6-\mathrm{mm}$ needle was introduced, $1 \mathrm{~cm}$ above the orbital rim, directed upward and laterally. A dose of 2-5 $\mathrm{U}$ was administered, according to the mass and strength of the muscle. At the intersection with the orbicularis, only the tip of the needle was introduced, directed upward and laterally, at least $1 \mathrm{~cm}$ from the orbital rim $(1-1.5 \mathrm{~cm})$. A dose of 1-2 $U$ was administered, according to the mass and strength of the muscle. ${ }^{20-22}$

\section{Procerus}

The injection point was placed in the intersection of the lines of the inner canthus and the opposite medial end of the eyebrow, coincidently with the medial facial line. In patients presenting a very strong procerus, a major fold was observed. In these cases, a second injection point was added, $1 \mathrm{~cm}$ above the first, on the medial facial line. A dose of 4-6 U was administered, according to the mass and strength of the muscle. The 6-mm needle was introduced a halfway deep, directed upward. ${ }^{20-22}$

\section{Depressor Supercilii}

The injection point was marked just below the medial end of the eyebrow, coincident with the horizontal line crossing the medial and lateral end of the brows, at least $1 \mathrm{~cm}$ from the orbital rim. Nearly $30 \%$ of the 6 -mm needle was introduced, directed medially and upward. A dose of 1-2 U was administered, according to the mass and strength of the muscle.

\section{Frontalis}

Two injection points were marked in the medial inferior part of the frontalis, an average of $2 \mathrm{~cm}$ above the eyebrows, coincident with the intersection of the lowest horizontal frown line on the forehead and the inner canthus line. Between $30 \%$ and $50 \%$ of the $6-\mathrm{mm}$ needle was introduced, directed upward. A dose of 1-2 $U$ was administered, according to the mass and strength of the muscle.

\section{Patient Assessment}

Standardized photos (frontal at rest and in maximum contraction) of the participants were blindly assessed by two experts to consensually grade the glabellar wrinkles (under maximum contraction) using the semiquantitative wrinklegrading MAS, a validated aesthetic grading scale to assess dynamic lines. The MAS scale graduates the wrinkles in maximum contraction on five levels ( 0 , no lines; 1 , mild lines; 2, moderate lines; 3 , severe lines; 4 , very severe lines). ${ }^{11}$

The patients were assessed on the day of injection (T0) and 4 weeks after treatment (T28); the standardized digital photographs taken during these visits were used for the blind assessment of MAS severity. Furthermore, the photos from $\mathrm{T} 0$ for the 5-point group were also blindly assessed by the muscular group involvement and asymmetry of the glabellar lines.

Efficacy was defined as an improvement of at least two points on the 5-point MAS for dynamic glabellar lines. This was performed to comply with a more stringent, recent recommendation by the US Food and Drug Administration. ${ }^{1}$ The primary outcome was the percentage of participants with a 2-point reduced MAS score for dynamic glabellar lines (under maximum contraction) at T28. ${ }^{11}$ Secondary outcomes were the total dose of BoNTA, the pattern of muscular recruitment for glabellar wrinkles, and the response rate according to the muscular group involvement and symmetry of the glabellar lines.

Adverse effects related to the treatments were assessed at T28. Demographic data (eg age and skin phototype) were also used for the analysis.

\section{Statistical Analysis}

The qualitative data was described as percentiles, and the proportions were compared through Pearson's chi-square test or chi-square test for trend. The 95\% confidence interval $(95 \% \mathrm{CI})$ from the percentiles was assessed through bootstrapping with 5000 resamples, with accelerated bias correction $(\mathrm{BCa})$. The quantitative data were reported as mean (standard deviation) or median (p25p75) if the normality was not confirmed by the Shapiro- 
Table I Baseline Severity (Glabellar Lines) and Demographic Data

\begin{tabular}{|c|c|c|c|c|}
\hline & 5-point & One2I & Total & p-value* \\
\hline $\mathrm{n}$ & 65 & 65 & 130 & \\
\hline Age (years) - mean (sd) & $46(9)$ & $44(8)$ & $45(9)$ & 0.162 \\
\hline Skin phototype - n (\%) & & & & 0.916 \\
\hline I & $13(20)$ & $16(25)$ & $29(22)$ & \\
\hline II & $31(48)$ & $24(37)$ & $55(42)$ & \\
\hline III & $17(26)$ & $23(35)$ & $40(31)$ & \\
\hline IV & $4(6)$ & $2(3)$ & $6(5)$ & \\
\hline MAS at baseline $-\mathrm{n}(\%)$ & & & & 0.578 \\
\hline Moderate & $24(37)$ & $27(42)$ & $51(39)$ & \\
\hline Severe & $34(52)$ & $33(50)$ & $67(52)$ & \\
\hline Very severe & $7(11)$ & $5(8)$ & $12(9)$ & \\
\hline
\end{tabular}

Notes: *Unadjusted p-value; MAS, Merz Aesthetics Scales for grading dynamic glabellar lines.

Wilk test ${ }^{23}$ the data were then compared between groups by a Student's $t$-test or Mann-Whitney U-test if indicated. The outcomes were compared according to time and the groups (over time) using generalized estimating equations (for ordinal data). ${ }^{24}$

The sample size was calculated to detect a difference greater than $15 \%$ between the groups regarding the reduction of $\geq 2$ points on the MAS at T28 (alpha $=0.05$; power $=0.8)^{25}$

Data were analyzed using the SPSS v25 software. Significance was set as $p<0.05$.

\section{Results}

Of the 149 patients enrolled in the study, 130 patients fulfilled the criteria (female sex and MAS score $\geq 2$ ) and were included in the analysis: 65 received the standard (5-point) treatment, and 65 underwent the One21 technique. The groups presented no substantial differences regarding demographics or baseline severity (Table 1).

According to the consensual blind assessment, the dynamic glabellar lines improved under maximum contraction at T28 in all participants. Overall, 116 patients (89.2\%; 95\% CI 84.6-93.8\%) presented at least a 2-point reduction in the MAS score. Of those patients, 64 (98.5\%; 95\% CI 96.9-99.9\%) were from the One21 group, and 52 $(80.0 \%$; $95 \%$ CI $70.8-87.7 \%)$ were from the 5-point group $(p<0.01)$.

When adjusted by age and phototype, both groups reduced the MAS score at T28 $(p<0.01)$, though the One21 group performed better $(p<0.01)$ than the 5 -point group (Figure 3). Both groups were homogeneous with respect to age and MAS score at $\mathrm{V} 1(\mathrm{p}=0.162$ and $\mathrm{p}=0578$, respectively).

Among patients enrolled in the 5-point group, the corrugators and procerus were the predominant muscles involved in the formation of glabellar dynamic lines; however, $83.1 \%$ of the participants also utilized other muscles (Table 2). Up to $60 \%$ of participants recruited depressor supercilii, up to $46 \%$ recruited orbicularis oculi, and up to $11 \%$ used the frontalis. Furthermore, 16 patients $(24.6 \%$; 95\% CI $14.5-35.3 \%$ ) presented lateral asymmetry in the glabellar lines (Figures 4 and 5).

The MAS score reduction in the 5-point group was lower for those with asymmetric glabellar lines $(\mathrm{OR}=$ 1.32; $p<0.01)$ and for those who utilized the

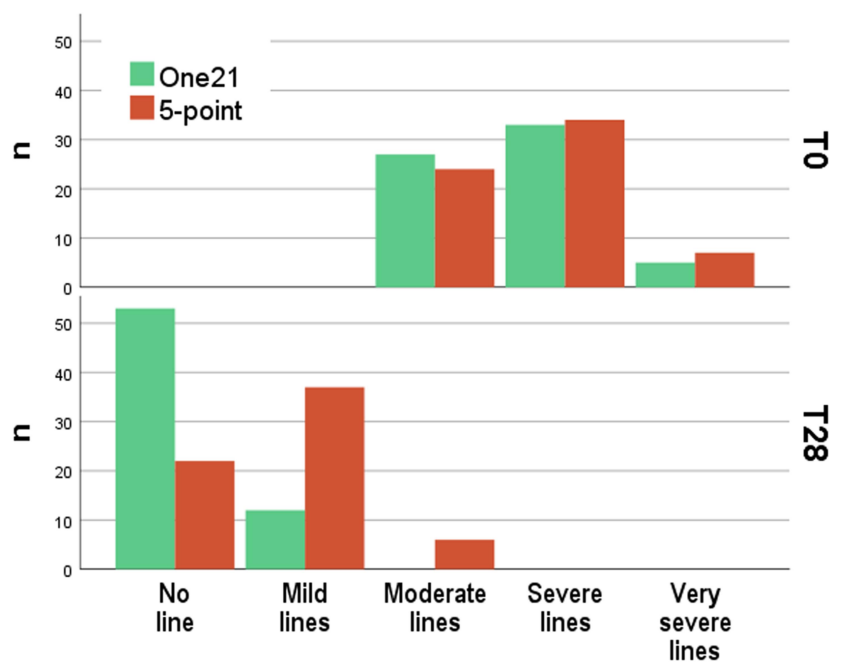

Figure 3 Merz Aesthetic Scales (MAS) scores for dynamic glabellar lines, before treatment (T0) and after four weeks (T28) for the One2I and standard 5-point groups $(n=130)$. 
Table 2 Muscular Group Involved in the Glabellar Lines Among Patients Who Underwent the 5-Point Regimen $(n=65)$

\begin{tabular}{|l|l|l|}
\hline Muscular Group & $\mathbf{n}$ & $\%$ \\
\hline Procerus & 64 & 98.5 \\
\hline $\begin{array}{l}\text { Corrugator } \\
\text { Left } \\
\text { Right }\end{array}$ & 65 & \\
\hline $\begin{array}{l}\text { Depressor supercilii } \\
\text { Left } \\
\text { Right }\end{array}$ & 65 & 100.0 \\
\hline Orbicularis & 40 & 100.0 \\
\hline Left & 39 & 61.5 \\
Right & & 60.0 \\
\hline Frontalis & 25 & 38.5 \\
\hline Lateral asymmetry & 30 & 46.2 \\
\hline
\end{tabular}

orbicularis $(\mathrm{OR}=1.23 ; p=0.02)$ and frontalis $(\mathrm{OR}=$ $1.23 ; p=0.04)$, but not for those who used the depressor supercilii $(\mathrm{OR}=0.96 ; p=0.68)$.

All but three patients from the 5-point group were treated with $20 \mathrm{U}$ of BoNT-A. The dose used in the One21 group was variable: mean (sd) $19.7 \mathrm{U}$ (2.9 U), and $20 \%$ were treated with up to $17 \mathrm{U}$ of BoNT-A.

The overall incidence of adverse effects in this series was low, and those effects were mild and transient. Headache and bruising were reported in $1.5 \%$ of patients, with no difference between the groups ( $p=$ 0.990). There was no eyelid ptosis, an adverse effect of special interest that was considered to be related to the treatment.
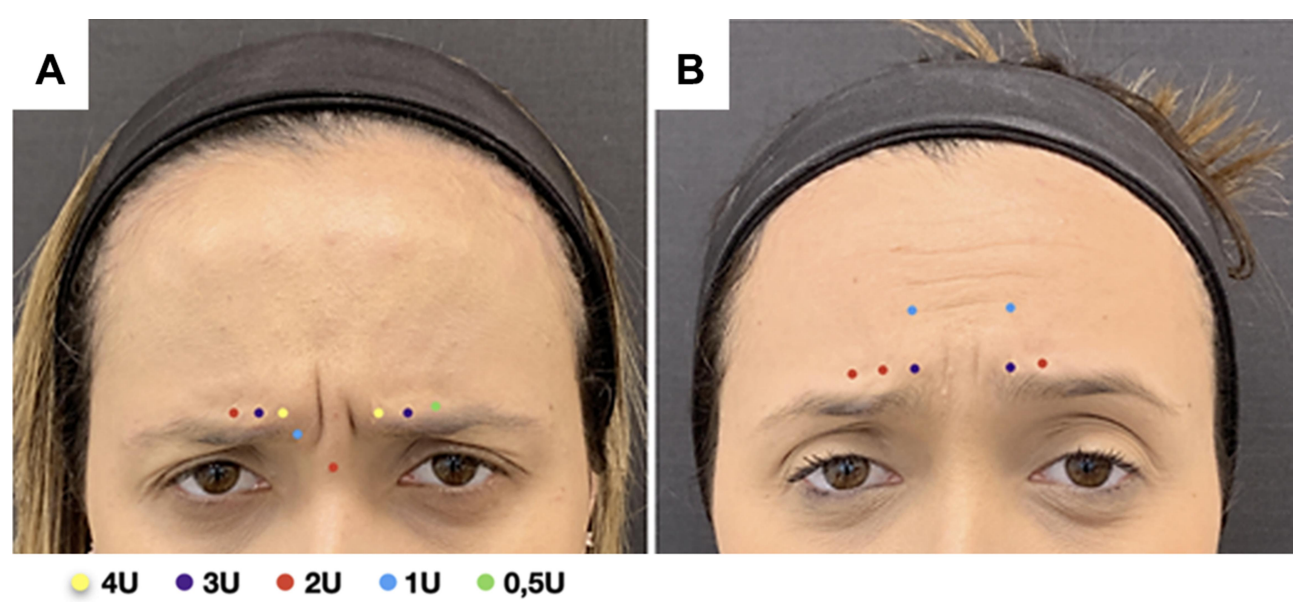

Figure 4 One2I assessment and personalized treatment plan based on glabellar line patterns. (A) Asymmetric glabellar lines: Prominence of right orbicularis and depressor supercilii. (B) Involvement of frontalis and right orbicularis in dynamic glabellar line formation.

\section{Discussion}

This study confirms incobotulinumtoxin-A is an effective and safe aesthetic treatment for glabellar dynamic lines. Furthermore, it suggests better performance is achieved through individualized assessment and treatment based on the anatomic references of the wrinkle formation.

The anatomy of the upper facial expression is complex, as the muscles are closely positioned and interact at different anatomical depths, creating a network of hyperdynamic skin lines that vary among individuals. ${ }^{26}$ In addition, eyebrow movements and glabellar lines are related to the dermal insertions of the muscle fibers, variations in weight, strength, muscle activity, and individual mimic patterns. ${ }^{9,26}$

The variability of the glabellar anatomy, associated with the behavioral characteristics of recruiting the muscular groups from this area, justifies an individual assessment and a personalized treatment regimen. In this series, patients treated with the One21 technique experienced a superior outcome, achieving significantly higher efficacy rates on Day 28 than those treated with the standard 5-point practice, with $98.5 \%$ and $80.0 \%$ of patients presenting $\geq 2$-point improvement in the MAS, respectively. In addition, patients with asymmetry in the glabellar contraction $(24.6 \%)$ treated with the 5-point technique had less successful results than those with symmetry.

The injection of botulinum toxin requires a proper understanding of the anatomical shape and insertion of the facial muscles to guide an objective muscle identification, resulting in a more effective approach. ${ }^{13,18}$ Sundaram et al assert that an inadequate number of injection points or 


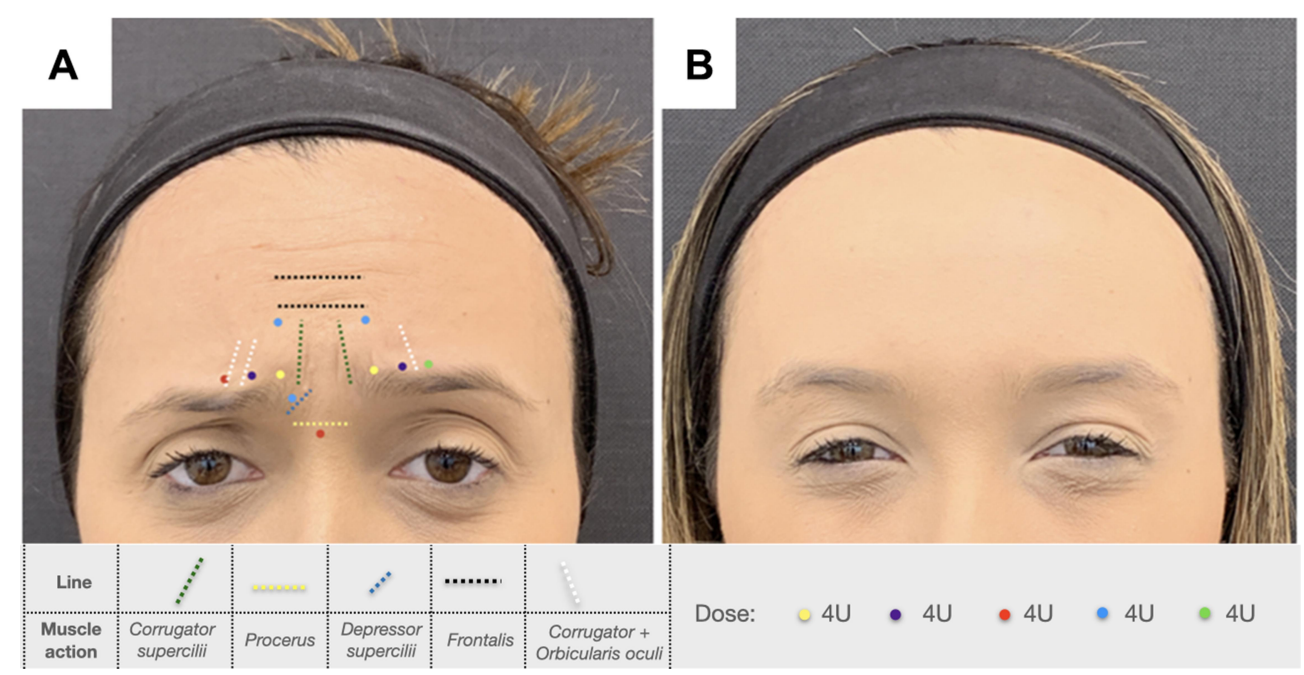

Figure 5 One2I assessment and personalized treatment plan based on glabellar line patterns. (A) Schematic mapping of the One2I technique for treating the procerus, corrugators, orbicularis oculi, and depressor supercilii. (B) Complete of glabellar frow wrinkles 28 days after the treatment.

doses can cause a partial or insufficient response to the application of the botulinum toxin. ${ }^{19}$ Knowledge of the targeted regional anatomy followed by precise injection technique that involves an adequate dose and depth of injection are essential not only to improve outcomes but also to avoid complications.

Previous clinical studies on the treatment of glabellar frown lines with neurotoxin have commonly considered an improvement of at least 1 point on the Facial Wrinkle Scale (FWS) or the MAS as the measure of the primary response to therapy, while others have required that patients achieve mild or no lines. ${ }^{10,27}$ The study published by Carruthers et al on the treatment of glabellar frown lines using the standard 5-point technique is one of the first to employ the newly mandated requirements of the FDA for a more robust definition of a successful response to the treatment, which requires an improvement of $\geq 2$ points on the FWS or MAS. ${ }^{28}$ In such trial, $76.6 \%$ of patients treated with $20 \mathrm{U}$ of incobotulinumtoxin-A (5 injection points; 4 U per point) had at least a 2-point improvement on the FWS on Day 30, according to the investigator assessment, ${ }^{28}$ a comparable rate of efficacy was demonstrated in our study in the 5-injection point group, with $80.0 \%$ of patients experiencing $\geq 2$-point improvement in the MAS for dynamic glabellar wrinkles on Day 28. However, in the group treated using the One21 technique, $98.5 \%$ of patients achieved this outcome, indicating a much superior performance compared to the standard 5-point approach.
In the current study, a reduction in the MAS score $\geq 2$ points for dynamic glabellar lines improvement was adopted as the primary efficacy, according to the blind assessments from photographs, following the rigorous new FDA criteria for assessing the efficacy of botulinum toxins. Another strength of our study is the severity wrinkle degree determined as the inclusion criteria, comprising moderate (2), severe (3) and very severe (4) frown lines in MAS for dynamic glabellar wrinkles. On Day $28,100 \%$ of the subjects from the ONE21 group, according to the investigator rating on MAS, scored none (0) or minimal wrinkles (1) at maximum frown, $81.5 \%$ and $18.5 \%$, respectively, reinforcing the effectiveness of the proposed technique.

The low incidence (1.5\%) of adverse effects relating to the treatment in this series confirms the safety of the 5-point and One 21 techniques. In the medical literature, the incidence of adverse effects is up to $3.2 \%$ in treatments with incobotulinumtoxin-A. ${ }^{22,27,28}$

Furthermore, patients from the One21 group were injected according to their individual needs considering dosage, targeted muscles, and point distribution. In the One21 group, the total dose of Incobotulinumtoxin-A per treatment ranged from 13 to $26 \mathrm{U}$ (mean $19.7 \mathrm{U}$ ), in alignment with the standard practice $(20 \mathrm{U})$. The importance of individualized treatment of the region was first emphasized by Almeida et al in categorizing five glabellar contraction patterns. Such a classification allows for a more accurate treatment of glabellar lines with botulinum toxin injection, resulting in more natural results. ${ }^{9}$ 
The One21 technique for injecting neurotoxin in the treatment of glabellar wrinkles focuses on an individualized assessment of the region that considers the influence of all brow depressor muscles and the functional anatomy in the interpretation of resultant dynamic lines. This technique enables the treatment of any pattern of glabellar wrinkles, including combined and asymmetric ones, optimizes the total dose distribution, and results in more effective outcomes (Figure 4).

This study has potential limitations related to being non-randomized; conversely, the groups were comparable regarding baseline characteristics, namely, age, sex, skin phototype and the degree of severity of the glabellar wrinkles baseline, data that positively strengthen our study.

A single evaluation at 4 weeks did not allow conclusions about the long-term efficacy of the One21 technique in comparison to the 5-point standard treatment, especially in those that received lower doses of BoNT-A. Further, specially designed prospective studies to assess the longterm efficacy of the One21 technique are warranted, including subject assessment of satisfaction and quality of life with the treatment of the glabellar lines.

\section{Conclusion}

Incobotulinumtoxin-A effectively reduced glabellar dynamic lines in all participants. Notably, the individualized assessment and treatment with the One 21 technique resulted in better performance achieving significantly higher efficacy rates than the standard 5-point treatment, in the treatment of the mild, moderate, severe glabellar wrinkles, especially for asymmetric lines of the glabella or the involvement of muscle groups other than the procerus and corrugator. Our results reinforce the importance of an individualized assessment and a customized treatment plan while injecting botulinum toxin A for the treatment of the glabellar wrinkles.

\section{Abbreviations}

BoNT-A, Botulinum toxin type A; FWS, Facial Wrinkle Scale; MAS, validated grading scale from Merz Aesthetics Scales for dynamic glabellar lines; T0, T28, visit at the inclusion and after 4 weeks.

\section{Acknowledgment}

The author would like to thank Dr. Birgit Bleßmann-Gurk for her input during manuscript revision and final approval.

\section{Disclosure}

Dr Carla de Sanctis Pecora reports non-financial support from Merz Aesthetics, during the conduct of the study; personal fees from Merz Aesthetics, outside the submitted work. Dr Maria Valéria Bussamara Pinheiro reports non-financial support from Merz Aesthetics, during the conduct of the study; personal fees from Merz Aesthetics, outside the submitted work. Dr Gisele Jacobino de Barros Nunes reports nonfinancial support from Merz, during the conduct of the study; personal fees from Merz, outside the submitted work Prof. Dr. Hélio Amante Miot reports personal fees from Merz Farmacêutica, during the conduct of the study. Carla Pecora, Gisele Jacobino de Barros Nunes, Maria Valéria Bussamara Pinheiro, and Karin Ventura Ferreira report being speakers and medical consultants for Merz Farmacêutica, SP, Brazil.

Hélio Miot reports being a medical writer and consultant for Merz Farmacêutica, SP, Brazil. The authors report no other potential conflicts of interest for this work.

\section{References}

1. Carruthers JD, Carruthers JA. Treatment of glabellar frown lines with C. botulinum-A exotoxin. J Dermatol Surg Oncol. 1992;18:17-21. doi:10.1111/j.1524-4725.1992.tb03295.x

2. Dorizas A, Krueger N, Sadick NS. Aesthetic uses of the botulinum toxin. Dermatol Clin. 2014;32:23-36. doi:10.1016/j.det.2013.09.009

3. Martinez AM. Visual perception of facial expressions of emotion. Curr Opin Psychol. 2017;17:27-33. doi:10.1016/j.copsyc.2017. 06.009

4. Cox SE, Finn JC. Social implications of hyperdynamic facial lines and patient satisfaction outcomes. Int Ophthalmol Clin. 2005;45:13-24. doi:10.1097/01.iio.0000167237.49396.7b

5. Miot HA, Penna GO, Ramos AMC, et al. Profile of dermatological consultations in Brazil (2018). An Bras Dermatol. 2018;93:916-928. doi:10.1590/abd1806-4841.20188802

6. Carruthers A, Carruthers J. Botulinum toxin type A: history and current cosmetic use in the upper face. Semin Cutan Med Surg. 2001;20:71-84. doi:10.1053/sder.2001.25138

7. Carruthers JD, Lowe NJ, Menter MA, Gibson J, Eadie N. Doubleblind, placebo-controlled study of the safety and efficacy of botulinum toxin type A for patients with glabellar lines. Plast Reconstr Surg. 2003;112:1089-1098. doi:10.1097/01.PRS.0000076504.7 9727.62

8. Carruthers JA, Lowe NJ, Menter MA, et al. A multicenter, double-blind, randomized, placebo-controlled study of the efficacy and safety of botulinum toxin type A in the treatment of glabellar lines. J Am Acad Dermatol. 2002;46:840-849. doi:10.1067/ mjd.2002.121356

9. de Almeida AR, da Costa Marques ER, Banegas R, Kadunc BV. Glabellar contraction patterns: a tool to optimize botulinum toxin treatment. Dermatol Surg. 2012;38:1506-1515. doi:10.1111/j.15244725.2012.02505.x

10. Sattler G, Callander MJ, Grablowitz D, et al. Noninferiority of incobotulinumtoxinA, free from complexing proteins, compared with another botulinum toxin type A in the treatment of glabellar frown lines. Dermatol Surg. 2010;36(Suppl 4):2146-2154. doi:10.1111/j.1524-4725.2010.01706.x 
11. Flynn TC, Carruthers A, Carruthers J, et al. Validated assessment scales for the upper face. Dermatol Surg. 2012;38:309-319. doi:10.1111/j.1524-4725.2011.02248.x

12. Rappl T, Parvizi D, Friedl H, et al. Onset and duration of effect of incobotulinumtoxinA, onabotulinumtoxinA, and abobotulinumtoxin A in the treatment of glabellar frown lines: a randomized, double-blind study. Clin Cosmet Investig Dermatol. 2013;6:211-219. doi:10.2147/ CCID.S41537

13. Ingallina F, Poulain B, Trevidic P. Anatomy \& Botulinum toxin injections.

14. Zarins U.

15. Cook BE Jr, Lucarelli MJ, Lemke BN. Depressor supercilii muscle: anatomy, histology, and cosmetic implications. Ophthalmic Plast Reconstr Surg. 2001;17:404-411. doi:10.1097/00002341-2001110 00-00004

16. Sykes JM, Trevidic P, Suarez GA, Criollo-Lamilla G. Newer understanding of specific anatomic targets in the aging face as applied to injectables: facial muscles-identifying optimal targets for neuromodulators. Plast Reconstr Surg. 2015;136:56S-61S. doi:10.1097/ PRS.0000000000001731

17. Trevidic P, Cauchois R, Ingallina F. [Facial anatomy and botulinum toxin: the muscular balances]. Ann Dermatol Venereol. 2009;136 (Suppl 4):S61-6. French. doi:10.1016/S0151-9638(09)74529-6

18. Abramo AC, Do Amaral TP, Lessio BP, De Lima GA. Anatomy of forehead, glabellar, nasal and orbital muscles, and their correlation with distinctive patterns of skin lines on the upper third of the face: reviewing concepts. Aesthetic Plast Surg. 2016;40:962-971. doi:10.1007/s00266-016-0712-z

19. Sundaram H, Signorini M, Liew S, et al. Global aesthetics consensus: botulinum toxin type a-evidence-based review, emerging concepts, and consensus recommendations for aesthetic use, including updates on complications. Plast Reconstr Surg. 2016;137:518e-29e. doi:10.1097/01.prs.0000475758.63709.23

20. Carruthers J, Fournier N, Kerscher M, Ruiz-Avila J, Trindade de Almeida AR, Kaeuper G. The convergence of medicine and neurotoxins: a focus on botulinum toxin type $\mathrm{A}$ and its application in aesthetic medicine-a global, evidence-based botulinum toxin consensus education initiative: part II: incorporating botulinum toxin into aesthetic clinical practice. Dermatol Surg. 2013;39:510-525. doi: $10.1111 /$ dsu. 12148
21. Yutskovskaya Y, Gubanova E, Khrustaleva I, et al. IncobotulinumtoxinA in aesthetics: russian multidisciplinary expert consensus recommendations. Clin Cosmet Investig Dermatol. 2015;8:297-306. doi:10.2147/CCID.S72301

22. de Maio M, Swift A, Signorini M, Fagien S. Facial assessment and injection guide for botulinum toxin and injectable hyaluronic acid fillers: focus on the upper face. Plast Reconstr Surg. 2017;140:265e76e. doi:10.1097/PRS.0000000000003544

23. Miot HA. Assessing normality of data in clinical and experimental trials. J Vasc Bras. 2017;16:88-91. doi:10.1590/1677-5449.041117

24. Miot HA. Análise de dados ordinais em estudos clínicos e experimentais. J Vascular Brasileiro. 2013;39:e20200185. doi:10.1590/1677-5449.200185

25. Miot HA. Sample size in clinical and experimental studies. J Vasc Bras. 2011;10:275-278. doi:10.1590/S1677-54492011000400001

26. Abramo AC. Muscle insertion and strength of the muscle contraction as guidelines to enhance duration of the botulinum toxin effect in the upper face. Aesthetic Plast Surg. 2018;42:1379-1387. doi:10.1007/ s00266-018-1157-3

27. Carruthers A, Carruthers J, Said S. Dose-ranging study of botulinum toxin type $\mathrm{A}$ in the treatment of glabellar rhytids in females. Dermatol Surg. 2005;31:414-22; discussion 22. doi:10.1097/ 00042728-200504000-00007

28. Carruthers A, Carruthers J, Coleman WP 3rd, et al. Multicenter, randomized, Phase III study of a single dose of incobotulinumtoxinA, free from complexing proteins, in the treatment of glabellar frown lines. Dermatol Surg. 2013;39:551-558. doi:10.1111/dsu. 12100
Clinical, Cosmetic and Investigational Dermatology

\section{Publish your work in this journal}

Clinical, Cosmetic and Investigational Dermatology is an international, peer-reviewed, open access, online journal that focuses on the latest clinical and experimental research in all aspects of skin disease and cosmetic interventions. This journal is indexed on CAS.
Dovepress

The manuscript management system is completely online and includes a very quick and fair peer-review system, which is all easy to use. Visit http://www.dovepress.com/testimonials.php to read real quotes from published authors. 